\title{
Biological Effects of and Responses to Exposure to Electrophilic Environmental Chemicals
}

\author{
Daigo Sumi* \\ Graduate School of Comprehensive Human Sciences, University of Tsukuba, 1-1-1 Tennodai, Tsukuba, Ibaraki 305-8575, Japan
}

(Received January 23, 2008)

\begin{abstract}
Electrophiles readily bind to nucleophilic centers on intracellular macromolecules such as DNA and proteins. Electrophilic attack on DNA results in the formation of an adduct, leading to depurination due to hydrolysis of a purine base such as adenine or guanine. On the other hand, electrophiles also attack proteins, with the thiolate function as the most attractive site. Many protein cysteine (Cys) thiols are affected by their proximity to basic amino acids, which results a decrease in the thiol pKa value. This paper discusses the role of electrophilenucleophile interactions in the adverse health effects of electrophilic environmental chemicals such as arsenic compounds (groundwater contaminant), methylmercury (MeHg; a fish contaminant), and 1,2-naphthoquinone (1,2-NQ; an atmospheric contaminant).
\end{abstract}

Key words — electrophile, arsenic, methylmercury, polycyclic aromatic hydrocarbon

\section{INTRODUCTION}

We are exposed to numerous chemical substances such as metals and polycyclic aromatic hydrocarbons (PAHs) that surround us in the environment. Because these chemicals are reported to have adverse health effects, electrophilic environmental chemicals have recently attracted considerable attention. When experimental animals and cultured cells are exposed to electrophiles, DNA and proteins are the molecular targets. Electrophiles react with DNA to form DNA-electrophile adducts that cause depurination of purine bases. When electrophiles react with proteins, their structure and function are affected, sometimes resulting in the unpredictable activation of cell signaling pathways and apoptosis (Fig. 1). Protective biological responses are also triggered by electrophiles to promote their detoxification. In this review, the biological responses to arsenic, methylmercury, and 1,2-naphthoquinone (1,2-NQ) as electrophilic environmental chemicals are discussed.

\footnotetext{
*To whom correspondence should be addressed: Graduate School of Comprehensive Human Sciences, University of Tsukuba, 1-1-1 Tennodai, Tsukuba, Ibaraki 305-8575, Japan. Tel.: +81-29-853-3297; Fax: +81-29-853-3297; E-mail: sdaigo@md.tsukuba.ac.jp
}

\section{CHARACTERISTICS OF PROTEINS VULNERABLE TO ELECTROPHILIC ENVIRONMENTAL CHEMICALS}

The dissociation of the cysteine (Cys) thiol group to its thiolate anion is required for the reaction with electrophiles. The $\mathrm{p} K a$ value of the thiol, clearly important in this interaction, can be markedly affected by adjacent basic amino acids in the secondary and tertiary structures of the protein. For example, the $\mathrm{pKa}$ value of Cys215 in protein tyrosine phosphatase 1B (PTP1B) is 5.4, ${ }^{1)}$ and that of Cys32 of thioredoxin (Trx) is 6.7.2) The low $\mathrm{pKa}$ values of these thiols make the corresponding proteins particularly vulnerable to electrophilic attack at physiological $\mathrm{pH}$ values. Although glutathione (GSH), a tripeptide with a Cys residue, exists at millimolar concentrations in the cells, the $\mathrm{pKa}$ value of its thiol is $8.66,{ }^{3)}$ and thus electrophiles can react with proteins such as PTP1B and Trx in spite of the higher GSH concentration.

\section{ARSENIC COMPOUNDS: GROUNDWATER CONTAMINANTS}

Arsenic compounds are ubiquitously distributed in nature. This metalloid forms a complex with 


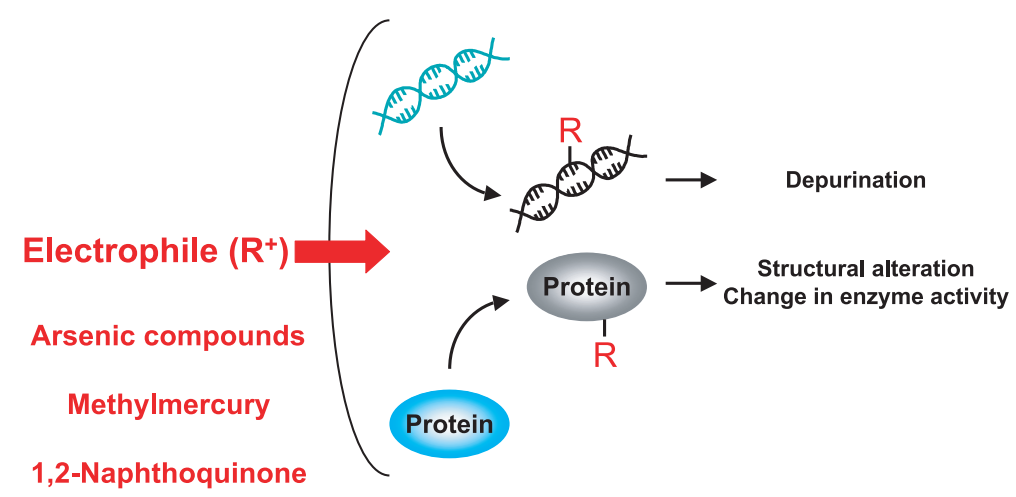

Fig. 1. Reactivity of Electrophilic Environmental Chemicals to DNA and Protein

pyrite. Under certain conditions, arsenic is dissociated from the complex and is found in groundwater. In East Asia, particularly Bangladesh, Vietnam, and China, more than 30 million people are chronically exposed to it. ${ }^{4)}$ Chronic arsenosis is associated with liver, skin, lung, and bladder cancer, hypertension, Raynaud's syndrome, and peripheral vascular disorders. Inorganic arsenite (iAsIII) is an electrophile and reacts with thiols, yielding monoand dithioarsenite. Once iAsIII incorporated into the cells through aquaporin 7 or 9, iAsIII is metabolized by repetitive reduction and oxidative methylation. Recently, Hayakawa et al. ${ }^{5)}$ have proposed a novel pathway in which GSH conjugation is an intermediate to methylation. Among the methylated arsenic metabolites, monomethylarsonous acid (MMeAsIII) is much more toxic and is suspected to be a stronger electrophile than inorganic arsenic. ${ }^{6)}$

Our group has found that arsenic exposure in cultured cells, experimental animals, and humans results in decreased levels of the endogenous vasodilator, nitric oxide (NO). ${ }^{7-9)}$ To determine the mechanisms for this decrease, we determined the effects of MMeAsIII on endothelial NO synthase (eNOS) activity. The results showed that MMeAsIII inhibits eNOS activity and that this inhibition is diminished in the presence of dithiothreitol, suggesting that the inhibitory action of MMeAsIII on eNOS occurs through covalent attachment to vicinal thiols of the protein. ${ }^{10)}$

Nuclear factor-erythroid 2-related factor 2 (Nrf2) is a basic leucine zipper transcription factor (bZip), which regulates the expression of phase II xenobiotic-metabolizing enzymes and phase III transporters. ${ }^{11-13)}$ Under normal conditions, Nrf2 is bound to Kelch-like ECH-associated protein 1 (Keap1) resulting in its ubiquitination. ${ }^{14)}$ Once the reactive thiols of Keap1 are modified by an electrophile, the hinge and latch model of the Nrf2/Keap1 binding motif is unbolted, leading to Nrf2 activation. ${ }^{15)}$ We found that iAsIII activates Nrf2 and upregulates antioxidant proteins such as heme oxygenase-1 (HO-1) and glutamylcysteine ligase (GCL) ${ }^{16)}$ We also studied the involvement of Nrf2 in iAsIII-induced cytotoxicity. With primary hepatocytes from wild-type or Nrf2-deficient mice, we demonstrated that iAsIII-induced cytotoxicity was greater in primary hepatocytes from Nrf2-deficient mice compared with wild-type hepatocytes. ${ }^{16)}$ Moreover, preincubation of the cells with sulphoraphane, an activator of Nrf2, prevented iAsIII-induced cytotoxicity through the reduction of arsenic accumulation in the cells. ${ }^{17}$ These results indicate that the Nrf2/Keap1 system is involved in the biological responses to arsenic exposure.

Diphenylarsinic acid (DPAsV) was found as a contaminant in well water in the Kamisu area of Ibaraki prefecture, Japan, and exposure through drinking the water led to difficulty in walking, tremors of the extremities, and memory disturbances. ${ }^{18)}$ We hypothesized that DPAsV could activate Nrf2 as well as iAsIII, thereby upregulating downstream proteins. Consistent with this notion, Nrf2 was activated, and HO-1 and the GCL modulatory subunit (GCLM) were upregulated during exposure of mouse primary hepatocytes to DPAsV. ${ }^{19)}$ $\mathrm{Nrf} 2$ is reported to upregulate the catalytic subunit of GCL (GCLC). However, DPAsV also cleaves the GCLC, resulting in decreased intracellular GSH levels through the activation of caspase-3. Caspase3 activation results from the p38 and/or JNK MAPK pathways, as DPAsV stimulates their phosphorylation. The data suggest that GCLC cleavage may impair the elimination of DPAsV, and the resulting intracellular accumulation may result in the apoptotic 
cell death of primary mouse hepatocytes. ${ }^{19)}$

\section{METHYLMERCURY (MeHg), A FISH CONTAMINANT}

In the environment, mercury $(\mathrm{Hg})$ comes from natural and anthropogenic sources, cinnabar, and combustion of fossil fuel. Once released to the oceans, rivers, and lakes, $\mathrm{Hg}$ is methylated to yield $\mathrm{MeHg}$ and dimethylmercury by aquatic organisms. $\mathrm{MeHg}$ is known to cause central nervous system dysfunction, because it readily penetrates the bloodbrain and placental barriers. ${ }^{20)} \mathrm{MeHg}$ accumulates in predatory fish species, and currently there is interest is in its health risk to humans who consume foodstuffs contaminated with $\mathrm{MeHg}$ such as tuna, swordfish, and shark.

$\mathrm{MeHg}$ exhibits a high affinity for sulfhydryl groups with a dissociation constant of $10^{-15},{ }^{21}$ ) and its association and dissociation reactions are rapid. ${ }^{22)}$ Upon cellular exposure, conjugates of protein sulfhydryl groups and the $\mathrm{MeHg}-\mathrm{GSH}$ conjugate are found. ${ }^{23,24)}$ The GSH conjugate is transported to the extracellular space through multidrugresistance proteins (MRPs). Among the MRPs, MRP1 and -2 are known to export GSH-conjugated substances. Since Nrf2 is negatively regulated by Cys thiols of the Keap1 protein, we hypothesized that MeHg might modify the Cys thiols of Keap1, leading to activation of Nrf2, which regulates MRP1 and -2 expression. Our rationale was that Nrf2 would play an important role in the $\mathrm{MeHg}$ excretion to the extracellular space. Consistent with this notion, primary hepatocytes from Nrf2-deficient mice were found to be susceptible to $\mathrm{MeHg}$-induced cytotoxicity, whereas liver-specific Keap1-deficient mice were resistant. ${ }^{25)}$ Under these conditions, intracellular $\mathrm{MeHg}$ was higher in Nrf2 deficiency and reduced in Keap1 deficiency with a concomitant change in MRP1 and -2 expression levels. ${ }^{25)}$ These findings suggest that the Nrf2/Keap1 system responds to $\mathrm{MeHg}$ exposure by stimulation of $\mathrm{MeHg}$ excretion.

\section{1,2-NQ, AN ATMOSPHERIC CONTAMINANT}

Exposure to diesel exhaust particles (DEP), which are part of the 2.5-micrometer particulate matter $\left(\mathrm{PM}_{2.5}\right)$ of ambient air, is associated with the induction of adverse health effects such as asthma, cancer, and cardiovascular disease. However, few studies have examined which component(s) causes these health effects. PAHs and their derivatives in DEP are among the substances thought to be potential candidates for these deleterious effects, and we have identified 1,2-NQ, as one of the quinones in ambient air samples. ${ }^{26)}$ 1,2-NQ possesses $\alpha, \beta$-unsaturated carbonyl groups and is a highly reactive electrophile that can react readily with protein thiolates. This property can be observed with 15-deoxy- $\Delta^{12,14}$-prostaglandin $\mathrm{J}_{2}\left(15 \mathrm{~d}-\mathrm{PGJ}_{2}\right)$, a metabolite of the prostaglandin cascade. $15 \mathrm{~d}$ $\mathrm{PGJ}_{2}$ reacts with the inhibitor of $\kappa \mathrm{B}$ kinase $\beta$ $(\mathrm{IKK} \beta), \mathrm{AP}-1$, and peroxisome proliferator-activated receptor $\gamma(\operatorname{PPAR} \gamma)$ to form covalent bonds, resulting in the alteration of their activities. ${ }^{27)}$ In previous studies, biotin-15d-PGJ 2 conjugate was used to demonstrate the binding of $15 \mathrm{~d}-\mathrm{PGJ}_{2}$ to protein. Based on those findings, we investigated whether $1,2-\mathrm{NQ}$ as an electrophile would bind covalently to $\mathrm{IKK} \beta$, cyclic adenosire $3^{\prime}, 5^{\prime}$-monophosphate (cAMP) response element-binding protein (CREB), and PTP1B, leading to the alteration of their activities. To address our hypothesis, we prepared a specific antibody against 1,2-NQ to detect proteins modified by this quinone.

The nuclear factor $-\kappa \mathrm{B}(\mathrm{NF}-\kappa \mathrm{B})$ signal is known to participate in numerous physiologic responses and it plays a crucial role in inflammatory responses. ${ }^{28)}$ Once lipopolysaccharide (LPS) and tumor necrosis factor interact with their receptors on the cell surface, the NF- $K \mathrm{~B}$ signaling cascade is activated, resulting in the upregulation of inflammatory cytokines, inducible NO synthase (iNOS), and cyclooxygenase-2. Exposure of RAW 264.7 cells to 1,2-NQ inhibited LPS-induced iNOS induction, NF- $\kappa \mathrm{B}$ activation, and inhibitor- $\kappa \mathrm{B}(\mathrm{I} \kappa \mathrm{B})$ phosphorylation. ${ }^{29)}$ An examination of the upstream kinase of NF- $\kappa \mathrm{B}, \mathrm{IKK} \beta$, verified that $1,2-\mathrm{NQ}$ covalently binds with $\operatorname{IKK} \beta$, thereby inhibiting its enzymatic activity. ${ }^{29)}$

CREB is a bZip, which plays an important role in cardiac function, tumor progression, and memory. bZip such as CREB and AP-1, contain highly conserved Cys residues that are crucial for DNA binding. ${ }^{30,31)}$ Because these Cys residues are surrounded by basic amino acids, their $\mathrm{pKa}$ values are likely to be relatively low, thereby becoming a molecular target for 1,2-NQ. In a study of the exposure of bovine aortic endothelial cells to $1,2-\mathrm{NQ}$, a 


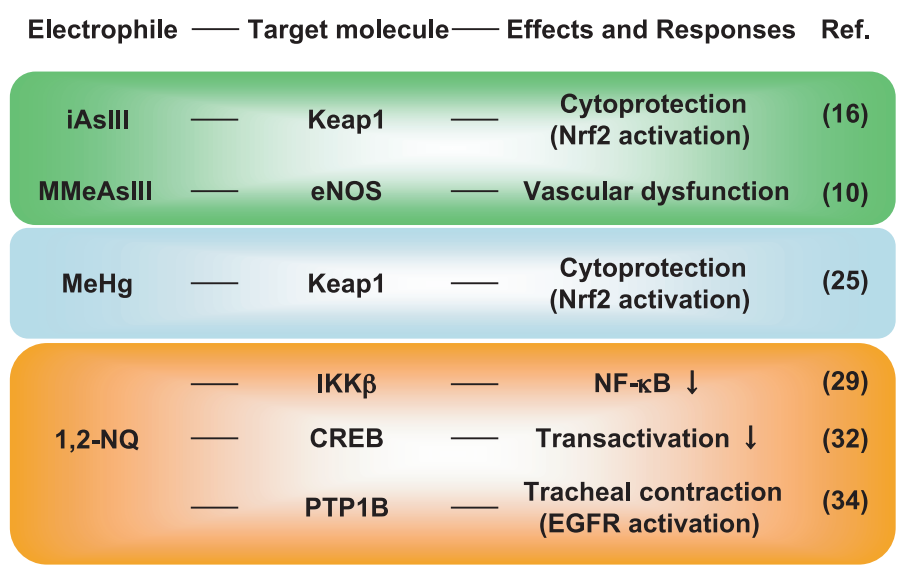

Fig. 2. Summary of This Review

loss of DNA binding and transcription activities as found, caused by the covalent binding of 1,2-NQ to CREB. ${ }^{32)}$

We have also examined 1,2-NQ effects on the guinea pig tracheal ring preparation and found that it induces a dose-dependent contraction. ${ }^{33)}$ The underlying mechanism for the contraction was found to be associated with the activation of epidermal growth factor receptor (EGFR) and vanilloid receptor (VR1) signaling. Thus pretreatment with an EGFR inhibitor, PD153035, restored 1,2-NQinduced tracheal contraction, indicating that contraction was, at least in part, attributable to EGFR phosphorylation that activates VR1, resulting in increased intracellular calcium content in the smooth muscle cells. ${ }^{33)}$ The dephosphorylation of tyrosine phosphates is as important as tyrosine phosphorylation in cell-signaling pathways. The protein tyrosine phosphatases (PTPs) catalyze the dephosphorylation of phosphotyrosine and one of them, PTP1B, negatively regulates EGFR activation by dephosphorylating the activated phosphotyrosine protein. The low $\mathrm{pK} a$ value of Cys215 in the active center of PTP1B ${ }^{1)}$ suggested that 1,2-NQ-induced EGFR activation may be the result of the inactivation of PTP1B through covalent bond formation. Results of studies investigating this possibility indicated that 1,2-NQ inhibits PTP activity in the cells through covalent modification. Experiments utilizing MALDI-TOF-MS/MS verified that histidine (His) 25 and Cys121 in PTP1B are targets for 1,2NQ. Subsequent analysis with site-directed mutagenesis showed that Cys121, but not His25, was partially responsible for the inhibition of PTP1B. ${ }^{34)}$

\section{CONCLUSIONS}

This review is summarized as shown in Fig. 2. eNOS, Nrf2/Keap1, IKK $\beta$, CREB, and PTP1B are target molecules of environmental electrophiles (arsenic, MeHg, and 1,2-NQ) with subsequent biological effects and responses. These phenomena will likely be observed with other environmental chemicals, such as cadmium and benzo[a]pyrene quinones, which have strong electrophilicity.

We are continuing our studies investigating additional proteins that are targets for electrophilic chemicals that result in cellular changes. To search for these targets, specific probes that can recognize electrophilic substances are useful, and we are undertaking experiments to identify electrophile chemical-bound proteins from the viewpoint of the thiolate anions of target molecules.

Acknowledgements I give my heartfelt thanks to Professor Yoshito Kumagai and laboratory members, both past and present, of the Department of Environmental Medicine, University of Tsukuba; Professor Masayuki Yamamoto of Tohoku University for providing Nrf2 and liver-specific Keap1deficient mice; and Professor Ken Itoh at Hirosaki University and Dr. Akira Yasutake of the National Institute for Minamata Disease for helpful advice. This work was supported in part by the Uehara Memorial Foundation, Sumitomo Foundation, and a grant-in-aid for Scientific Research from the Ministry of Education, Science, Culture and Sports of Japan (\#18790376). 


\section{REFERENCES}

1) Lohse, D. L., Denu, J. M., Santoro, N. and Dixon, J. E. (1997) Roles of aspartic acid-181 and serine222 in intermediate formation and hydrolysis of the mammalian protein-tyrosine-phosphatase PTP1. Biochemistry, 36, 4568-4575.

2) Li, H., Hanson, C., Fuchs, J. A., Woodward, C. and Thomas, G. J., Jr. (1993) Determination of the pKa values of active-center cysteines, cysteines-32 and -35, in Escherichia coli thioredoxin by Raman spectroscopy. Biochemistry, 32, 5800-5808.

3) Srinivasan, U., Mieyal, P. A. and Mieyal, J. J. (1997) $\mathrm{pH}$ profiles indicative of rate-limiting nucleophilic displacement in thioltransferase catalysis. Biochemistry, 36, 3199-3206.

4) Nordstrom, D. K. (2002) Public health. Worldwide occurrences of arsenic in ground water. Science, 296, 2143-2145.

5) Hayakawa, T., Kobayashi, Y., Cui, X. and Hirano, S. (2005) A new metabolic pathway of arsenite: arsenic-glutathione complexes are substrates for human arsenic methyltransferase Cyt19. Arch. Toxicol., 79, 183-191.

6) Styblo, M., Drobna, Z., Jaspers, I., Lin, S. and Thomas, D. J. (2002) The role of biomethylation in toxicity and carcinogenicity of arsenic: a research update. Environ. Health Perspect., 110 (Suppl 5), 767-771.

7) Pi, J., Horiguchi, S., Sun, Y., Nikaido, M., Shimojo, N., Hayashi, T., Yamauchi, H., Itoh, K., Yamamoto, M., Sun, G., Waalkes, M. P. and Kumagai, Y. (2003) A potential mechanism for the impairment of nitric oxide formation caused by prolonged oral exposure to arsenate in rabbits. Free Radic. Biol. Med., 35, 102-113.

8) Pi, J., Kumagai, Y., Sun, G., Yamauchi, H., Yoshida, T., Iso, H., Endo, A., Yu, L., Yuki, K., Miyauchi, T. and Shimojo, N. (2000) Decreased serum concentrations of nitric oxide metabolites among Chinese in an endemic area of chronic arsenic poisoning in inner Mongolia. Free Radic. Biol. Med., 28, 11371142.

9) Kumagai, Y. and Pi, J. (2004) Molecular basis for arsenic-induced alteration in nitric oxide production and oxidative stress: implication of endothelial dysfunction. Toxicol. Appl. Pharmacol., 198, 450-457.

10) Sumi, D., Taguchi, K., Sun, Y., Shinkai, Y. and Kumagai, Y. (2005) Monomethylarsonous acid inhibits endothelial nitric oxide synthase. J. Health Sci., 51, 728-730.

11) Hayashi, A., Suzuki, H., Itoh, K., Yamamoto, M. and Sugiyama, Y. (2003) Transcription factor Nrf2 is required for the constitutive and inducible expression of multidrug resistance-associated protein 1 in mouse embryo fibroblasts. Biochem. Biophys. Res. Commun., 310, 824-829.

12) Itoh, K., Chiba, T., Takahashi, S., Ishii, T., Igarashi, K., Katoh, Y., Oyake, T., Hayashi, N., Satoh, K., Hatayama, I., Yamamoto, M. and Nabeshima, Y. (1997) An Nrf2/small Maf heterodimer mediates the induction of phase II detoxifying enzyme genes through antioxidant response elements. Biochem. Biophys. Res. Commun., 236, 313-322.

13) Vollrath, V., Wielandt, A. M., Iruretagoyena, M. and Chianale, J. (2006) Role of Nrf2 in the regulation of the Mrp2 (ABCC2) gene. Biochem. J., 395, 599609.

14) Itoh, K., Wakabayashi, N., Katoh, Y., Ishii, T., Igarashi, K., Engel, J. D. and Yamamoto, M. (1999) Keap1 represses nuclear activation of antioxidant responsive elements by Nrf2 through binding to the amino-terminal Neh2 domain. Genes Dev., 13, 7686.

15) Tong, K. I., Padmanabhan, B., Kobayashi, A., Shang, C., Hirotsu, Y., Yokoyama, S. and Yamamoto, M. (2007) Different electrostatic potentials define ETGE and DLG motifs as hinge and latch in oxidative stress response. Mol. Cell. Biol., 27, 7511-7521.

16) Kumagai, Y. and Sumi, D. (2007) Arsenic: signal transduction, transcription factor, and biotransformation involved in cellular response and toxicity. Annu. Rev. Pharmacol. Toxicol., 47, 243-262.

17) Shinkai, Y., Sumi, D., Fukami, I., Ishii, T. and Kumagai, Y. (2006) Sulforaphane, an activator of $\mathrm{Nrf2}$, suppresses cellular accumulation of arsenic and its cytotoxicity in primary mouse hepatocytes. FEBS Lett., 580, 1771-1774.

18) Ishii, K., Tamaoka, A., Otsuka, F., Iwasaki, N., Shin, K., Matsui, A., Rudo, G., Kumagai, Y., Ishii, T., Shoji, S., Ogata, T., Ishizaki, M., Doi, M. and Shimojo, N. (2004) Diphenylarsinic acid poisoning from chemical weapons in Kamisu, Japan. Ann. Neurol., 56, 741-745.

19) Sumi, D., Manji, A., Shinkai, Y., Toyama, T. and Kumagai, Y. (2007) Activation of the Nrf2 pathway, but decreased gamma-glutamylcysteine synthetase heavy subunit chain levels and caspase-3-dependent apoptosis during exposure of primary mouse hepatocytes to diphenylarsinic acid. Toxicol. Appl. Pharmacol., 223, 218-224.

20) Simmons-Willis, T. A., Koh, A. S., Clarkson, T. W. and Ballatori, N. (2002) Transport of a neurotoxicant by molecular mimicry: the methylmercury-Lcysteine complex is a substrate for human L-type 
large neutral amino acid transporter (LAT) 1 and LAT2. Biochem. J., 367, 239-246.

21) Simpson, R. B. (1961) Assosiation constants of methylmercury with sulfhydryl and other bases. $J$. Am. Chem. Soc., 83, 4711-4717.

22) Carty, A. J. and Malone, S. F. (1979) The chemistry of mercury in biological systems. In The Biogeochemistry of Mercury in the Environment (Nriagu, J. O., Ed.), Elsevier, Amsterdam, pp. 433-470.

23) Clarkson, T. W. (1997) The toxicology of mercury. Crit. Rev. Clin. Lab. Sci., 34, 369-403.

24) Fujiyama, J., Hirayama, K. and Yasutake, A. (1994) Mechanism of methylmercury efflux from cultured astrocytes. Biochem. Pharmacol., 47, 1525-1530.

25) Toyama, T., Sumi, D., Shinkai, Y., Yasutake, A., Taguchi, K., Tong, K. I., Yamamoto, M. and Kumagai, Y. (2007) Cytoprotective role of Nrf2/Keap1 system in methylmercury toxicity. Biochem. Biophys. Res. Commun., 363, 645-650.

26) Cho, A. K., Stefano, E. D., You, Y., Rodriguez, C. E., Schmitz, D. A., Kumagai, Y., Miguel, A. H., Eiguren-Fernandez, A., Kobayashi, T., Avol, E. and Fronines, J. R. (2004) Determination of four quinones in diesel exhaust particles, SRM 1649a and atmospheric $\mathrm{PM}_{2.5}$. Aerosol Sci. Technol., 38, 1-14.

27) Kim, E. H. and Surh, Y. J. (2006) 15-deoxyDelta12,14-prostaglandin J2 as a potential endogenous regulator of redox-sensitive transcription factors. Biochem. Pharmacol., 72, 1516-1528.

28) Hayden, M. S., West, A. P. and Ghosh, S. (2006) NF-kappaB and the immune response. Oncogene,
25, 6758-6780.

29) Sumi, D. and Kumagai, Y. (2007) Chemical biology of 1,2-naphthoquinone, a novel air pollutant, that affects signal transduction pathways. Yakugaku Zasshi, 127, 1949-1956 (in Japanese).

30) Abate, C., Patel, L., Rauscher, F. J., III and Curran, T. (1990) Redox regulation of fos and jun DNA-binding activity in vitro. Science, 249, 11571161.

31) Vinson, C., Acharya, A. and Taparowsky, E. J. (2006) Deciphering B-ZIP transcription factor interactions in vitro and in vivo. Biochim. Biophys. Acta., 1759, 4-12.

32) Endo, A., Sumi, D. and Kumagai, Y. C. (2007) 1,2-Naphthoquinone disrupts the function of cAMP response element-binding protein through covalent modification. Biochem. Biophys. Res. Commun., 361, 243-248.

33) Kikuno, S., Taguchi, K., Iwamoto, N., Yamano, S., Cho, A. K., Froines, J. R. and Kumagai, Y. (2006) 1,2-Naphthoquinone activates vanilloid receptor 1 through increased protein tyrosine phosphorylation, leading to contraction of guinea pig trachea. Toxicol. Appl. Pharmacol., 210, 47-54.

34) Iwamoto, N., Sumi, D., Ishii, T., Uchida, K., Cho, A. K., Froines, J. R. and Kumagai, Y. (2007) Chemical knockdown of protein-tyrosine phosphatase 1B by 1,2-naphthoquinone through covalent modification causes persistent transactivation of epidermal growth factor receptor. J. Biol. Chem., 282, 3339633404. 